\title{
August 2014 Tucson Critical Care Journal Club: Bacteremia in Cardiac Arrest
}

\section{Article: Coba V, Jaehne AK, Suarez A, Dagher GA, Brown SC, Yang JJ, et al. The incidence and significance of bacteremia in out of hospital cardiac arrest. Resuscitation. 2014;85:196-202. [CrossRef] [PubMed]}

Out-of-hospital cardiac arrest (OHCA) is an uncommon, but important, condition encountered in the emergency department (ED). While cardiac arrest represents the final common pathway of multiple conditions, early evaluation often focuses on cardiac abnormalities. However, observed associations between infection, particularly pneumonia, and in-hospital cardiac arrest led Coba et al. (1) to investigate the incidence of bacteremia among OHCA patients.

The study prospectively investigated 250 adult patients who presented to an academic ED with OHCA between 2007 and 2009. Two blood culture samples were drawn during resuscitation or shortly after return of spontaneous circulation through vascular devices placed for clinical purposes. Children, pregnant women, victims of trauma were excluded. To minimize false positive results, patients were classified as bacteremic if one sample was positive for a typical pathogen or both samples were positive for the same skin colonizing organism. Patients in whom only 1 sample was positive for suspected skin contaminant or in whom no growth was observed after 5 days were classified as non-bacteremic.

Of 173 patients, 65 (38\%) were considered to have bacteremia preceding the cardiac arrest. Bacteremic and non-bacteremic patients were similar along measured demographic and clinical characteristics at presentation. No differences in CPR duration, location of arrest, or presenting rhythm were noted. Few patients survived to hospital discharge, $3 \%$ of those classified as bacteremic versus $5 \%$ of those classified as non-bacteremic $(p=0.66)$. While no differences in survival were observed at 28 days or at hospital discharge, survival to hospital admission was lower among bacteremic patients as compared to non-bacteremic patients, $25 \%$ versus $40 \%$, respectively $(p=0.04)$.

Among patients who survived to hospital admission, a higher proportion of bacteremic, as opposed to non-bacteremic, patients were vasopressor dependent, $93.8 \%$ vs $74.4 \%$, respectively ( $p=.06)$. Furthermore, bacteremic patients had higher lactate levels $(p=0.01)$, lower arterial $p H(p=0.01)$, and higher potassium $(p<0.01), B U N(p=0.02)$, creatinine $(p=0.02)$, magnesium $(p=0.01)$, and phosphorous $(p=0.01)$ levels. Bacteremic patients were also more likely to have antibiotics started in the ED, $69 \%$ versus $30 \%$, respectively $(p=0.01)$. Cultures yielded a wide range of bacterial species with streptococcal and staphylococcal species being the most frequently observed. Up to $40 \%$ of patients presenting to the ED with OHCA appear to have clinically significant bacteremia. Given that bacteremic patients were twice as likely to receive antibiotics in the ED as compared to those without bacteremia, the treating clinicians appeared to frequently recognize this possibility. Compared to those without confirmed bacteremia, those with bacteremia had physiologic abnormalities that were consistent 
with severe sepsis including higher lactate levels and greater metabolic acidosis. While long-term outcomes remain poor, clinicians should still consider and appropriately treat co-existing infection when caring for cardiac arrest patients in the ED.

Additional research is needed to determine the clinical relevance of bacteremia in this setting. An important remaining question is to what extent infection precipitates cardiac arrest versus being an incidental finding in the immediate post-arrest period due to transient bacteremia caused by resuscitation efforts and/or translation of bacterial across poorly perfused gut. Given the high proportion of bacteremic patients receiving antibiotics, examination of other data (e.g., urinalysis, chest radiography, etc.) might have helped identify a plausible foci of infection.

The low survival to discharge observed in this study, $<5 \%$, would tend to hide any treatment benefit that might have accrued to patients in whom early treatment of severe sepsis was initiated. Future study in a setting with higher survival to discharge would be needed to determine whether treatment of co-existing infection in cardiac arrest patients might lead to improved survival.

Cameron Hypes MD MPH ${ }^{1,2}$, Joe K. Gerald, MD, $\mathrm{PhD}^{3}$

${ }^{1}$ Department of Medicine, Division of Pulmonary, Allergy, Critical Care, and Sleep Medicine, University of Arizona Medical Center; Tucson, AZ

${ }^{2}$ Department of Emergency Medicine, University of Arizona Medical Center; Tucson, AZ

${ }^{3}$ College of Public Health, University of Arizona Medical Center; Tucson, AZ

\section{References}

1. Coba V, Jaehne AK, Suarez A, Dagher GA, Brown SC, Yang JJ, et al. The incidence and significance of bacteremia in out of hospital cardiac arrest. Resuscitation. 2014;85:196-202. [CrossRef] [PubMed]

2. Neumar RW, Otto CW, Link MS, Kronick SL, Shuster M, Callaway CW, et al. Part 8: Adult advanced cardiovascular life support: 2010 american heart association guidelines for cardiopulmonary resuscitation and emergency cardiovascular care. Circulation. 2010;122:S729-S767. [CrossRef] [PubMed]

3. Nichol G, Thomas E, Callaway CW, Hedges J, Powell JL, Aufderheide TP, et al. Regional variation in out-of-hospital cardiac arrest incidence and outcome. JAMA. 2008;300:1423-31. [CrossRef] [PubMed]

4. Dunne RB, Compton S, Zalenski RJ, Swor R, Welch R, Bock BF. Outcomes from out-of-hospital cardiac arrest in detroit. Resuscitation. 2007;72:59-65. [CrossRef] [PubMed] 